\title{
Mindfulness for vertigo and tinnitus
}

Volume 4 Issue 3 - 2016

\section{Opinion}

Mindfulness is a skill that can be taught in clinic with vertigo and tinnitus patients. I saw the immediate benefits in patients who asked for these skills when I worked at The University of Melbourne Balance Disorders Clinic. This was a diagnostic service where we spent up to 3-hours performing tests on each patient. We had plenty of time to practice mindfulness skills between each little diagnostic procedure.

These days, I run a clinic that is devoted to teaching these rehabilitation skills and supporting patients through the day-to-day reality of living with vertigo, dizziness or tinnitus.

My patients report that since practising mindfulness, they have:

a. Improved sleep;

b. Greater self-confidence;

c. Better connection with their body (legs, hips, feet);

d. Improved stability;

e. A greater sense of calm and relaxation in daily life;

f. And an improved ability to "bounce back" when symptoms are more severe.

I have seen the benefits unfold in literally thousands of people. It is humbling. Mindfulness is a skill that anyone can learn. Research has shown that the practice of mindfulness can rewire our brain and change the shape of our neural firing patterns. This is a big deal. Our brains form a large part of who we are and how we experience the world. Our brains are important for the recovery of vertigo, dizziness and tinnitus.

Mindfulness helps us to better focus and choose where we place our attention. Our attention feeds brain activity. Brain activity shapes how we see, feel and hear the world around and within us. As we practice skills and tools that improve our focus, we can better choose where we put our attention. We are armed with the power of choice. This can be challenging in the learning stages. And liberating once we get the hang of it.

If you have experienced vertigo, dizziness or tinnitus, then you know- it isn't fun! Many patients report loss of confidence and worry about their future. Will I need to quit my job? Will this get better? Why is this happening to me? Many patients don't know what has changed or why things have changed. But their body and their sensations have changed.

It can happen overnight. Some people report hearing roaring, buzzing, ringing, popping or sliding sounds within their ears, head or body. They don't know why it's suddenly noisy, but it is. They are now distracted by a cacophony of noise in their every moment of life.

It is there. It is still there. It is exhausting. No-one else can see it. No-one else can hear it some patients report that it feels intolerable, beyond frustration. They feel isolated and stuck.Some patients want to escape; but they can't leave their body and they can't escape this

\author{
Joey Remenyi \\ Department of Vestibular Audiologist, Melbourne University, \\ Australia
}

Correspondence: Joey Remenyi, Department of Vestibular Audiologist Mindfulness Educator Seeking Balance, Geelong, Melbourne University, Australia,

Email info@seekingbalance.com.au

Received: June 22, 2016 | Published: July 01, 2016

sound. It is still there. I have had times when I could hear my own tinnitus very loudly too. It was distressing. It was difficult. I certainly needed tools to help me through it.

I practiced mindfulness. It transformed my approach to recovery. In clinic, some dizzy patients report feeling like their brain is swimming inside their head; or feeling less confident in daily life. This 'dizzy' feeling is unwanted. It is unpleasant. It is unfamiliar. It can seem interminable. Vertigo, dizziness and tinnitus can occur at any age or stage in our lives. It is never fun and it is never easy. But you can get through it. Your body wants to find its balance again. This process is known as 'compensation', 'adaptation' or 'the process of recovery'. Sometimes it happens with an inner ear change or trauma. Other times these symptoms occur when we are in shock or rushing around in daily life and low on blood sugar!

Either way, it doesn't feel safe, pleasant or good. For some, symptoms naturally resolve and disappear with time. For others, their tinnitus sounds and dizzy sensations can persist and become worse. This can lead to anxiety, depression and feelings of profound hopelessness and frustration. Understandably too. These impacts on our ability to see, feel and trust the world around us.

Mindfulness has been a great gift for my patients who want support through these feelings of frustration and uncertainty. In clinic we sit together and begin to practice specific exercises that help us contact a sense of safety, connection and clarity. During each moment, with or without symptoms, we learn to genuinely feel okay.

We learn how to choose our point of focus and make space for feelings of dizziness or sounds of tinnitus. We learn how to preserve and protect our precious life energy, rather than spending it fighting unwanted feelings and trying to get rid of them. We learn how to feel whole and okay, just as we are right there in clinic or in each moment of our day. We learn and practice these skills and tools together. Tools for life. Sounds pretty good, doesn't it?

Mindfulness skills are a practice. They teach us to connect with what really matters to us deep within our hearts. A mindfulness practice offers us support through difficult times. It supports the repair process within our biology as we relax into our body, and observe it with curiosity. Our 'fight and flight' feelings are reduced as we choose not to struggle. We refocus and reconnect. We offer self-kindness in place of judgment and everything "suddenly" feels okay. 
For Free Resources visit Seekingbalance.com.au

For a list of research articles and pdfs related to this topic visit http://www.seekingbalance.com.au/research-articles/

\section{Acknowledgments}

None.

\section{Conflicts of interest}

Author declares there are no conflicts of interest.

Funding

None. 